\title{
Etude des variations de rendement quantique interne d'un détecteur CCD en fonction de la température
}

\author{
G. Rolland \\ CNES, Centre Spatial de Toulouse, 18, avenue Edouard Belin, 31055 Toulouse Cedex, France
}

(Reçu le 6 juillet 1983, révisé le 28 janvier 1985, accepté le 21 mai 1985)

\begin{abstract}
Résumé. - A partir du relevé des courbes de réponses spectrales d'une barrette photosensible à transfert de charges à différentes températures entre 280 et $78 \mathrm{~K}$, par ajustement à un modèle théorique du rendement interne d'un photoélément, nous dégageons les paramètres dominants qui déterminent l'évolution du rendement interne de la partie photosensible avec la température. Nous trouvons que ces paramètres sont la longueur de diffusion dans la zone avant du photoélément et le coefficient d'absorption du matériau. Nous donnons les lois de variations de ces deux paramètres.
\end{abstract}

\begin{abstract}
The mean parameters which control the variations of the internal quantum efficiency of the active layer with temperature are determined from the spectral response curves of a CCD photosensitive array. The measurements are operated in a large temperature range (from $78 \mathrm{~K}$ to $280 \mathrm{~K}$ ). We optimize a theoretical model and by fitting it with experimental data we evaluate critical parameters. We find that these parameters are the diffusion length in the front layer and the intrinsic absorption coefficient of the silicon substrate. The variation laws of these two parameters are given.
\end{abstract}

\section{Introduction.}

L'exposé qui suit est relatif à l'observation des variations du rendement quantique interne d'une barrette photosensible à transfert de charges en fonction de la température. De la comparaison des résultats expérimentaux et des prévisions d'un modèle théorique $\mathrm{du}$ rendement interne du photoélément, nous dégageons les paramètres dominants qui déterminent l'évolution du rendement de la zone active avec la température.

Le paragraphe 2 contient la définition du rendement interne et le choix des modèles théoriques.

Le paragraphe 3 décrit brièvement le dispositif expérimental et les conditions de mesure adoptées. La suite du texte expose le résultat des observations relatives aux variations de réponse spectrale (\$ 4); la méthode, les résultats et les interprétations des ajustements.

\section{Modélisation de la zone photosensible.}

2.1 RAPPEL DU FONCTIONNEMENT GLOBAL DE LA BARRETTE. - La séquence de fonctionnement de ces dispositifs peut être schématisée comme suit (voir Fig. 1). Une ligne de 1728 photoéléments indépendants

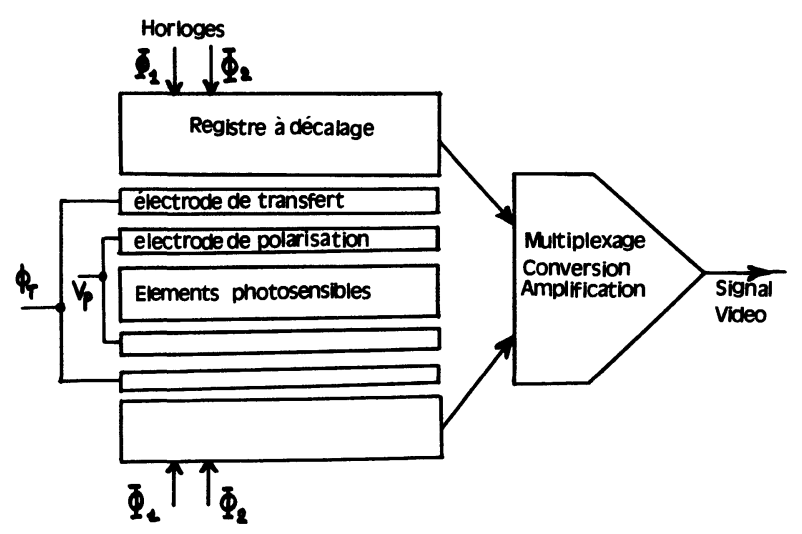

Fig. 1. - Structure de la barrette photosensible à CCD étudiée.

[Charge coupled device structure.]

intègre pendant une durée $T_{0}$ appelée « temps d'intégration " un signal lumineux. Le flux de photons tombant sur le photoélément $\mathrm{n}^{\circ} j$ pendant le temps d'intégration est converti en un paquet d'électrons. Ce paquet de charges est localisé à la fin du temps d'intégration dans la zone de stockage associée à l'élément $n^{\circ} j$. 
Au bout du temps $T_{0}$, une électrode de transfert commande le chargement parallèle des informations contenues dans la ligne photosensible dans deux registres à décalage analogiques biphasés à canal enterré.

Le temps de chargement des registres est très inférieur au temps d'intégration (quelques centaines de ns devant quelques $\mathrm{ms}$ ).

Un des registres est chargé par le contenu des photoéléments de rang pair et l'autre par celui des photoéléments de rang impair.

Quand le chargement parallèle des deux registres est terminé, l'électrode $\phi_{\mathrm{T}}$ isole à nouveau les photoéléments des deux registres et un nouveau temps d'intégration commence.

Pendant que les photoéléments intègrent une nouvelle ligne d'image, les registres à décalage véhiculent vers les étages de sortie les informations qu'ils contiennent.

Le train des 1728 informations correspondant à une ligne d'image est reformé par multiplexage des sorties des registres et amplifié.

Pour le détail du fonctionnement des registres et des étages de sortie, on pourra se reporter, par exemple, aux références $[1,3]$.

\section{2 FONCTIONNEMENT DE LA ZONE PHOTOSENSIBLE. -} Le détail de la structure de la zone photosensible et l'ordre de grandeur de ses dimensions sont donnés sur les figures $2 a$ et $2 b$.

On voit qu'un photoélément est constitué par l'association d'une jonction $\mathrm{n}^{+} / \mathrm{p}$ et d'une grille de stockage. La zone $\mathrm{n}^{+}$est délimitée par des caissons d'isolement $\left(\mathrm{Si}-\mathrm{O}_{2}\right)$ et une implantation $\mathrm{p}^{+}$. La grille de stockage, polarisée positivement par rapport au fond du substrat, impose la polarisation inverse de la jonction $\mathrm{n}^{+} / \mathrm{p}$. Le flux de photons entrant par la fenêtre optique crée, dans la jonction $\mathrm{n}^{+} / \mathrm{p}$, un photocourant qui vient charger la capacité MOS formée par la grille de stockage, le $\mathrm{SiO}_{2}$ et le substrat $\mathrm{P}$. Au bout du temps d'intégration, on dispose sous la grille de stockage d'un nombre de charges fonction des caractéristiques du flux de photons. Le paquet de charges sera transformé en échantillon de tension par l'étage de conversion-amplification (Fig. 1).

\subsection{CHOIX DU MODÈLE DE RENDEMENT QUANTIQUE.}

2.3.1 Définition de la réponse spectrale. - Pour une radiation monochromatique de longueur d'onde $\lambda$, en appelant $E_{j}$ la valeur moyenne de la densité de puissance du faisceau vue par le photoélément $n^{\circ} j$ pendant le temps d'intégration $T_{0}$ et $V_{\mathrm{S} j}$ l'échantillon de tension associé à la réponse de ce même photoélément, on définit la réponse spectrale $R_{j}$ par la quantité :

$$
R_{j}(\lambda)=\frac{V_{\mathrm{S} j}}{T_{0} \cdot E_{j}}
$$

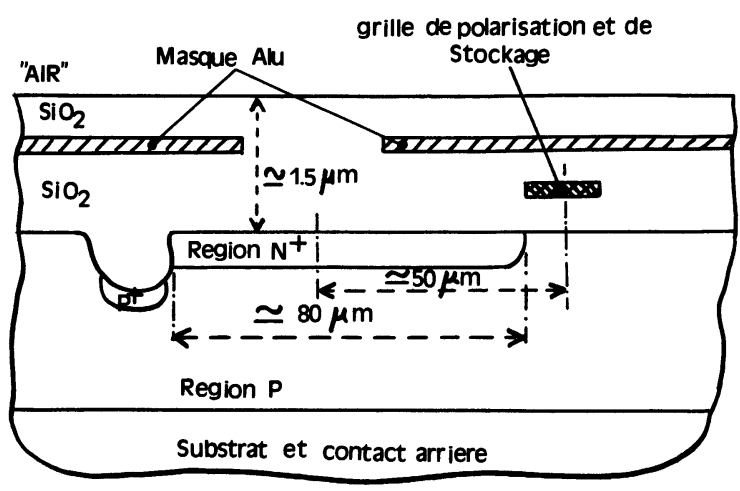

Fig. 2a. - Structure détaillée de la zone photosensible (coupe suivant l'axe AA de la figure $2 b$ ).

[Photosensitive area structure (Cross section view).]

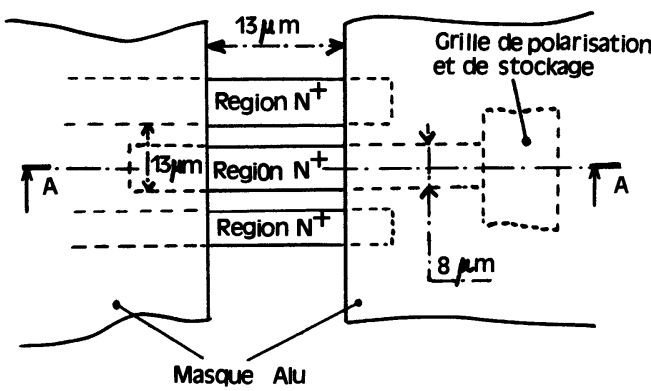

Fig. 2b. - Vue de dessus, d'une partie de la ligne photosensible.

[Photosensitive structure (Top view).]

$R_{j}(\lambda)$ se déduit directement des relevés expérimentaux de $V_{\mathrm{Sj}}, E_{j}$ et $T_{0}$.

2.3.2 Rendement quantique externe d'un photoélément. - Le rendement quantique externe $\eta_{\mathrm{e}}$ de l'élément photosensible est défini par le rapport du nombre d'électrons collectés dans le paquet de charges au nombre de photons incidents pendant le temps d'intégration.

Si $q$ est la valeur absolue de la charge de l'électron, $\frac{h c}{\lambda}$ l'énergie d'un photon de longueur d'onde $\lambda, S$ l'aire de la surface sensible et $N_{j}$ le nombre d'électrons collectés dans le paquet de charges, on a :

$$
q N_{j}=\frac{E_{j} \cdot T_{0} \cdot S}{\left(\frac{h c}{q \lambda}\right)} \eta_{\mathrm{e} j}
$$

En notant $k$ le coefficient reliant la variation de la tension de sortie $V_{\mathrm{s} j}$ de l'élément $j$ à la charge totale $\mathrm{du}$ paquet d'électrons collectés : $V_{\mathrm{Sj}}=k q N_{j}$, on obtient :

$$
V_{\mathrm{S} j}=\frac{E_{j} \cdot T_{0}}{\left(\frac{h c}{q \lambda}\right)} k S \eta_{\mathrm{e} j}
$$


qu'on réécrit sous la forme (en utilisant (1)) :

$$
\eta_{\mathrm{e} j}=\frac{\left(\frac{h c}{q \lambda}\right)}{k S} R_{j}(\lambda) .
$$

L'expression (2) relie le rendement quantique externe de l'élément $\mathrm{n}^{0} j$ à la valeur de la réponse spectrale.

\subsubsection{Rendement quantique interne d'un photoélé-} ment. - Si on définit le rendement quantique interne $\eta_{\mathrm{i}}$ par le rapport du nombre d'électrons collectés dans le paquet de charges au nombre de photons traversant la surface inférieure du $\mathrm{SiO}_{2}$ à la verticale de l'élément actif, on a, en notant $t(\lambda)$ la transmission globale de l'ensemble air- $\mathrm{SiO}_{2}$ :

$$
\eta_{\mathrm{e}}=t(\lambda) \eta_{\mathrm{i}}
$$

La relation entre le rendement interne de l'élément photosensible et sa réponse spectrale s'écrit :

$$
\eta_{\mathrm{ij}}(\lambda)=\left(\frac{h c}{q S k}\right) \frac{R_{j}(\lambda)}{\lambda t(\lambda)} .
$$

2.3.4 Identification au modèle unidimensionnel du rendement interne d'une jonction abrupte. - Nous cherchons à définir un modèle théorique du rendement interne de l'élément photosensible faisant intervenir les paramètres caractéristiques des phénomènes de transport dans les différentes parties de la zone active.

Dans l'hypothèse où la recombinaison des électrons sur le trajet allant du $\mathrm{n}^{+}$à la zone de stockage est négligeable, le rendement interne du photoélément peut être identifié à celui de la jonction verticale $\mathrm{n}^{+} / \mathrm{p}$.

Le calcul de l'expression générale donnant le rendement quantique interne théorique de cette jonction doit tenir compte de l'existence d'interactions entre photoéléments voisins (diaphotie).

Cet effet est spécifique aux radiations allant du rouge à l'infrarouge proche $(0,7$ à $1,1 \mu \mathrm{m})$ et peut être résumé comme suit :

- pour ce domaine de longueurs d'onde, le faisceau incident au photoélément $\mathrm{n}^{\mathrm{o}} j$ génère des paires électron-trou en profondeur dans le substrat $\mathbf{P}$; les électrons diffusent dans la partie $P$ et ont une probabilité non nulle d'être happés par les zones de charges d'espace des photoéléments de rang $j \pm n$.

On voit, de ce fait, que la valeur du rendement interne d'un élément photosensible peut dépendre de l'éclairement reçu par les photoéléments voisins.

Nous verrons cependant (cf. §3.2.1) qu'il est possible de choisir des conditions expérimentales d'éclairement telles que l'incidence de la diaphotie sur la valeur du rendement interne d'un photoélément donné puisse être négligée. Ces conditions sont telles que les charges perdues par un photoélément soient compensées par l'apport des éléments voisins; en d'autres termes, les courants horizontaux sous ce photoélément se compensent. Par conséquent, dans ces conditions, l'expression du rendement interne du photoélément étudié coïncide avec celle du rendement interne d'une jonction plane unidimensionnelle.

Ce rendement interne est égal au rapport de la densité du photocourant (ramenée à la surface éclairée) au courant de photons entrant à la verticale de la surface active éclairée. Soit (avec les notations précédentes) :

$$
\eta_{\mathrm{i} j}=\frac{\left(\frac{N_{j}}{S . T_{0}}\right)}{\frac{E_{j} t(\lambda)}{\left(\frac{h c}{\lambda}\right)}}=\frac{J_{\mathrm{p}}}{J_{\mathrm{ph}}}
$$

(où $J_{\mathrm{p}}$ et $J_{\mathrm{ph}}$ sont respectivement les densités de photocourant et de courant de photons d'une jonction plane unidimensionnelle).

\subsubsection{Rappel de l'expression donnant le rendement} interne d'une jonction plane unidimensionnelle. - Le calcul est effectué à partir de la structure simplifiée donnée sur la figure 3 .

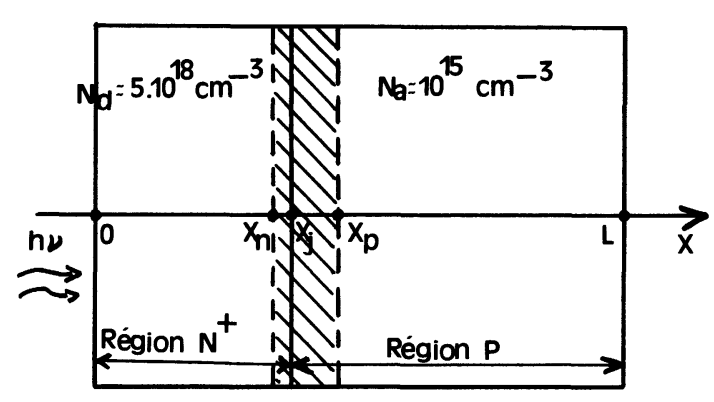

Fig. 3. - Définition des dimensions intervenant dans le modèle unidimensionnel.

[Characteristic dimensions of the unidimensional model.]

On se place dans le cadre de l'approximation de la jonction abrupte : dopage uniforme dans les parties $\mathrm{n}^{+}$ et $p$ avec saut dans le plan de la jonction. On suppose qu'en $x=0$, la photodiode reçoit un éclairement stationnaire, monochromatique à rayons parallèles et perpendiculaires au plan d'abscisse $x=0$ et que ce rayonnement est absorbé dans le matériau suivant la loi classique $E(x)=E(0) \mathrm{e}^{-\alpha x}$.

$E(x)$ désigne la densité de puissance du faisceau à l'abscisse $x$ et $\alpha$ est le coefficient d'absorption intrinsèque du silicium. Le calcul des photocourants circulant dans la jonction se fait à partir de l'équation de continuité (Réf. [5] p. 307).

Nous appelons $D_{\mathrm{p}}$ le coefficient de diffusion des trous dans le $\mathrm{n}^{+}, S_{\mathrm{a}}$ la vitesse de recombinaison des trous dans le plan de cote $x=0, L_{\mathrm{p}}$ la longueur de diffusion des trous dans le $\mathrm{n}^{+}$et $D_{\mathrm{n}}, L_{\mathrm{n}}$ les quantités 
correspondantes pour les électrons de la partie $\mathbf{P}$. $\mathrm{La}$ résolution de l'équation de continuité avec les conditions aux limites (5),

$$
\begin{aligned}
& \hat{n}(L)=0 \\
& \hat{n}\left(x_{\mathrm{p}}\right)=0 \\
& \hat{p}\left(x_{\mathrm{n}}\right)=0
\end{aligned}
$$

$$
S_{\mathrm{a}} \hat{p}(0)=\left.D_{\mathrm{p}} \frac{\partial \hat{p}}{\partial x}\right|_{(x=0)}
$$

(où $\hat{n}$ et $\hat{p}$ sont les densités de porteurs minoritaires en excès par rapport à l'équilibre induites par l'éclairement) conduit à la valeur du rendement quantique interne (Réfs. [7, 4]) :

$$
\left.\begin{array}{c}
\eta_{\mathrm{i}}=\eta_{\mathrm{n}^{+}}+\eta_{\mathrm{ZD}}+\eta_{\mathrm{p}} \quad\left(L_{\mathrm{s}}=\frac{D_{\mathrm{p}}}{S_{\mathrm{a}}}\right) \\
\eta_{\mathrm{n}^{+}}=\frac{\left(\alpha L_{\mathrm{p}}\right)^{2}}{1-\left(\alpha L_{\mathrm{p}}\right)^{2}}\left\{\mathrm{e}^{-\alpha x_{\mathrm{n}}}-\frac{1+\alpha L_{\mathrm{s}}}{\alpha L_{\mathrm{p}}\left[\sinh \left(\frac{x_{\mathrm{n}}}{L_{\mathrm{p}}}\right)+\frac{L_{\mathrm{s}}}{L_{\mathrm{p}}} \cosh \left(\frac{x_{\mathrm{n}}}{L_{\mathrm{p}}}\right)\right]}+\frac{\mathrm{e}^{\alpha x_{\mathrm{n}}}\left[\frac{L_{\mathrm{p}}}{L_{\mathrm{s}}} \cosh \left(\frac{x_{\mathrm{n}}}{L_{\mathrm{p}}}\right)+\sinh \left(\frac{x_{\mathrm{n}}}{L_{\mathrm{p}}}\right)\right]}{\alpha L_{\mathrm{p}}\left[\frac{L_{\mathrm{p}}}{L_{\mathrm{s}}} \sinh \left(\frac{x_{\mathrm{n}}}{L_{\mathrm{p}}}\right)+\cosh \left(\frac{x_{\mathrm{n}}}{L_{\mathrm{p}}}\right)\right]}\right\} \\
\eta_{\mathrm{ZD}}=\mathrm{e}^{-\alpha x_{\mathrm{n}}}-\mathrm{e}^{-\alpha x_{\mathrm{p}}} \\
\eta_{\mathrm{p}}=\frac{\left(\alpha L_{\mathrm{n}}\right)^{2}}{\left(\alpha L_{\mathrm{n}}\right)^{2}-1}\left\{\mathrm{e}^{-\alpha x_{\mathrm{p}}}+\frac{\mathrm{e}^{-\alpha L}-\mathrm{e}^{-\alpha x_{\mathrm{p}}} \cosh \left(\frac{L-x_{\mathrm{p}}}{L_{\mathrm{n}}}\right)}{\alpha L_{\mathrm{n}} \sinh \left(\frac{L-x_{\mathrm{p}}}{L_{\mathrm{n}}}\right)}\right.
\end{array}\right\}
$$

où $\eta_{\mathrm{n}^{+}}, \eta_{\mathrm{ZD}}$ et $\eta_{\mathrm{p}}$ sont les contributions respectives de la partie $\mathbf{n}^{+}$, de la zone de charge d'espace et de la zone $\mathbf{P}$ au rendement interne global $\eta_{\mathrm{i}}$. Cette expression du rendement interne est valable dans le cas où la densité de porteurs en excès par rapport à l'équilibre est faible (condition que nous supposerons vérifée dans tout ce qui suit).

Dans le calcul des expressions (6), on suppose, de plus, implicitement que la structure de bande dans la zone $\mathrm{n}^{+}$est la même que celle de la zone $\mathrm{P}$. Nous supposons donc que les corrections à apporter aux équations de transport (Réf. [6]) du fait de l'important dopage de la zone avant sont négligeables. La valeur de $\alpha$ en fonction de $\lambda$ et de la température est donnée au $\S 2.4$.

Les coordonnées $x_{n}$ et $x_{p}$ des limites de la zone de charge d'espace sont calculées pour une polarisation de $-4 \mathrm{~V}$ et à chaque température par les formules classiques relatives aux jonctions abruptes (Réf. [3] pages 75 et 77).

En résumé, dans le cadre des hypothèses énumérées ci-dessus et pour des conditions expérimentales données telles que l'incidence de la diaphotie puisse être négligée, nous identifierons le rendement quantique interne du photoélément (défini par l'expression (3)) au rendement interne d'une jonction abrupte plane unidimensionnelle (équations (6)).

\subsection{VARIATION DU COEFFICIENT D'ABSORPTION AVEC} LA TEMPÉRATURE POUR LES LONGUEURS D'ONDE COMPRISES ENTRE 0,5 ET $1 \mu \mathrm{m}$. - Nous supposerons que, pour le photoélément considéré, de tous les modes d'absorption, répertoriés (Réf. [10] page 561), seule est significative l'absorption intrinsèque et, de plus, que l'effet des contraintes internes du matériau est négligeable. Les seules données expérimentales que nous connaissons, relatives au coefficient d'absorption intrinsèque du silicium en fonction de la température, sont celles de Dash et Newman (Réf. [11]). Ces auteurs donnent les courbes d'absorption à deux températures : $300 \mathrm{~K}$ et $77 \mathrm{~K}$ pour un monocristal de silicium. Nous avons adapté l'expression théorique donnant la valeur de $\alpha$ en fonction de l'énergie du photon et de la température (Réf. [5] page 263) de telle sorte que les valeurs calculées soient en accord avec les données expérimentales de Dash et Newman dans tout le domaine spectral qui nous concerne.

Dans le domaine des énergies de photons allant de 1,2 à $2,5 \mathrm{eV}$, notre valeur de $\alpha$ s'écrit :

- Pour des énergies de photon comprises entre 1,2 et $2,2 \mathrm{eV}$ :

$$
\begin{aligned}
& \begin{aligned}
& \alpha=2750\left\{\begin{array}{r}
\frac{\left(E-1,1+5 \times 10^{-7} T^{2}\right)^{2}}{\exp \left(\frac{348}{T}\right)-1}+ \\
\quad+\frac{\left(E-1,16+5 \times 10^{-7} T^{2}\right)^{2}}{1-\exp \left(-\frac{348}{T}\right)}
\end{array}\right\} \\
& \quad-\text { Et entre } 2,2 \mathrm{et} 2,5 \mathrm{eV}:
\end{aligned} \mid \\
& \alpha=\alpha(2,2 \mathrm{eV} ; T)\left\{\frac{7447,3}{\alpha(2,2 \mathrm{eV} ; T)}+0,4491\right\}^{\left(\frac{E}{0,3}-7,3333\right)}
\end{aligned}
$$


$\alpha$ est donné en $\mathrm{cm}^{-1} ; E$ en électron-volts et $T$ en Kelvins.

Ces expressions tiennent compte des variations de largeur de bande interdite avec la température (Réf. [5] page 71). Sur la figure 4, sont tracées les courbes $\alpha(E, T)$.

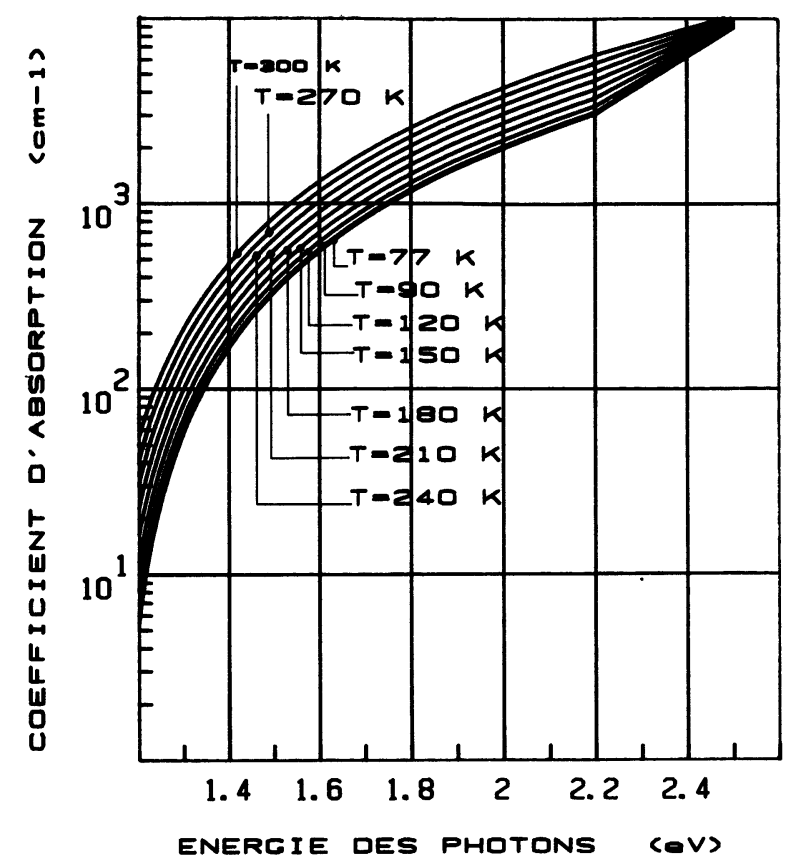

Fig. 4. - Variations du coefficient d'absorption avec l'énergie des photons et la température tracées à partir de la relation (7).

[Variations of absorption coefficient versus photon energy and temperature deduced from equation (7).]

En l'absence de toute autre donnée expérimentale, nous serons conduits dans ce qui suit à supposer que les valeurs de $\alpha$ aux températures comprises entre 300 et $77 \mathrm{~K}$ sont correctement décrites par (7).

\section{Dispositif expérimental et conditions de mesure.}

\subsection{DisPOSITIF EXPÉRIMENTAL (FIG. 5).}

3.1.1 Optique de projection des taches lumineuses et mesure $d u$ flux. - Un monochromateur à double prisme sélectionne à partir d'une source de lumière blanche une radiation monochromatique dont la longueur d'onde centrale peut être ajustée de façon continue entre $0,33 \mu \mathrm{m}$ et $1,2 \mu \mathrm{m}$ avec une largeur de bande à mi-hauteur comprise (pour nos essais) entre 3 et $12 \mathrm{~nm}$.

Le faisceau de sortie du monochromateur est repris par une optique et projeté à travers la fenêtre en quartz du cryostat sur le CCD. Les dimensions de la tache lumineuse sont fixées par un diaphragme. Une partie du faisceau est dérivée en amont de l'optique de projection vers un détecteur étalon (photodiode).

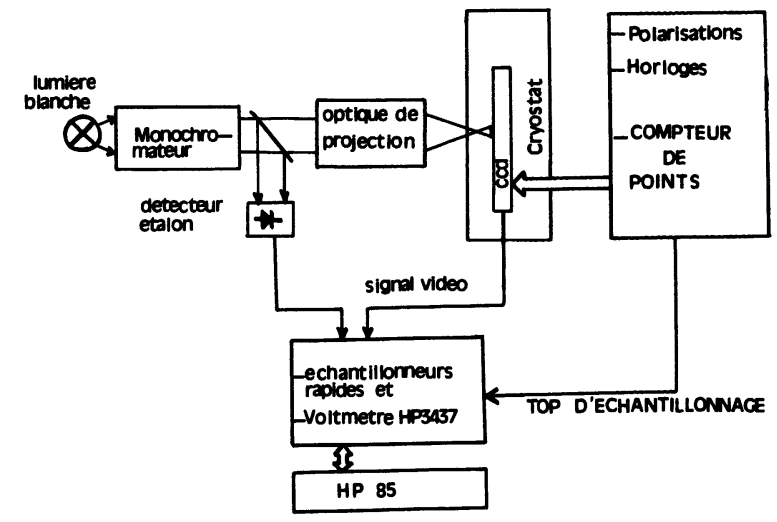

Fig. 5. - Schéma du dispositif expérimental.

[Experimental measurements system.]

Une table d'étalonnage et un sous-programme de correction permettent au calculateur de relier la valeur du signal de sortie du détecteur étalonné au flux lumineux réellement reçu par le CCD.

3.1.2 Contrôle de la température du composant. Le détecteur CCD est en contact avec un doigt froid placé à l'intérieur d'une enceinte où on effectue un vide de l'ordre de $10^{-4}$.

Une pompe établit un courant constant d'azote liquide à travers le circuit de refroidissement. Une résistance électrique et un thermocouple incorporés au doigt contrôlent la température de manière à l'asservir à une consigne manuelle ou à variation programmée.

3.1.3 Signaux de contrôle. - Une série d'alimentations stabilisées réalise l'ensemble des polarisations continues du composant.

Une logique appropriée, sous le contrôle de l'horloge principale, génère l'ensemble des phases de commande du détecteur CCD. Ce même bloc effectue le repérage du rang de l'échantillon de signal de sortie à analyser et envoie à la section d'acquisition l'ordre d'échantillonnage au moment où cet échantillon se présente en sortie du composant.

3.1.4 Section d'acquisition et de traitement. - Le signal de sortie du CCD est traité suivant le principe du double échantillonnage (Réfs. $[8,7])$ et lu par un voltmètre rapide interfacé avec un minicalculateur HP85. Ce même voltmètre est commuté sur le signal de sortie du détecteur étalon à chaque mesure de l'amplitude du flux lumineux.

Un logiciel assure la gestion des opérations et la programmation du voltmètre, le contrôle de la table traçante et les opérations de traitement des données définies au $\S 5$.

\subsection{CONDITIONS DE MESURE.}

3.2.1 Conditions d'éclairement. - Le relevé du profil d'interaction typique d'un élément avec ses voisins 
est donné sur la figure 6. Le relevé est effectué en éclairant le photoélément de rang $j$ avec une tache circulaire de $6 \mu \mathrm{m}$ de diamètre placée au centre de sa surface active. On voit qu'au-delà de l'élément de rang $j \pm 6$, la réponse des photoéléments en obscurité est inférieure au pour cent de la valeur de la réponse de l'élément éclairé.

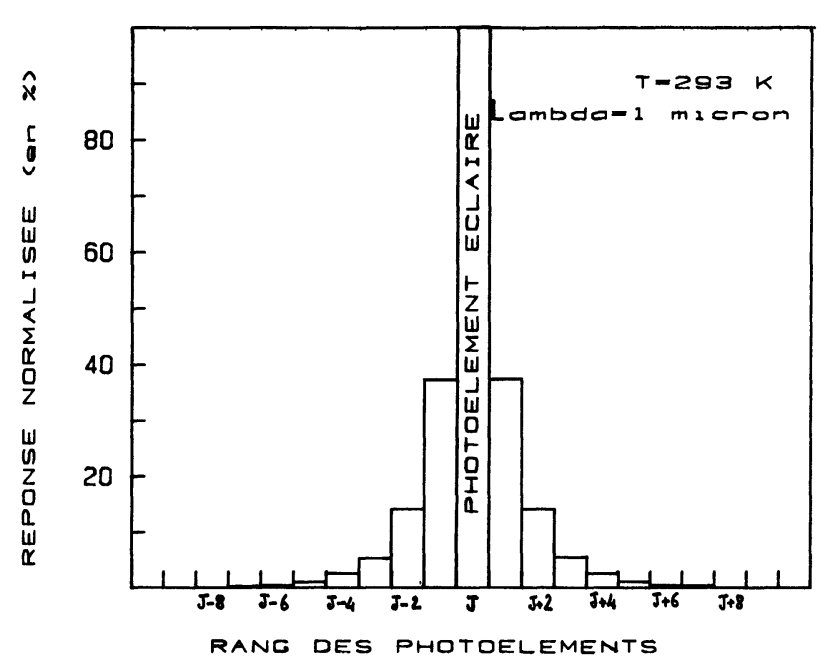

Fig. 6. - Profil d'interaction entre éléments photosensibles à $\lambda=1 \mu \mathrm{m}$ et $T=293 \mathrm{~K}$.

[Typical cross talk between photosites at $\lambda=1 \mu \mathrm{m}$ and $T=293 \mathrm{~K}$.]

On a donc choisi d'éclairer, pendant nos mesures de réponse spectrale, la ligne photosensible avec une tache rectangulaire telle que dix éléments de part et d'autre de l'élément analysé reçoivent un flux identique.

Dans de telles conditions d'éclairement, on voit que la quantité de charges perdue par diaphotie par l'élément $j$ est compensée par l'apport des éléments voisins si le profil d'interaction d'un élément avec ses voisins est identique pour tous les éléments éclairés. (Ce dernier point est vérifié expérimentalement au moment du choix de la zone analysée.)

3.2.2 Mesures des valeurs de $R_{j}(\lambda)$. - Pour chaque longueur d'onde $\lambda$, la chaîne d'acquisition relève :

- le niveau moyen d'obscurité de l'élément $j$ : $\left\langle V_{\text {jobsc }}\right\rangle$

- le niveau moyen de sortie en éclairement stationnaire de l'élément $j:\left\langle V_{j \text { ecl. }}\right\rangle$

- le niveau moyen de sortie du détecteur étalon : $\left\langle V_{\text {ph }}\right\rangle$.

Les moyennes sont prises sur 400 échantillons de rang $j$ pris sur des temps d'intégration consécutifs.

La durée du temps d'intégration est fixée à $T_{0}=3 \mathrm{~ms}$. Quand les trois moyennes précédentes ont été calculées, le HP85 donne la valeur de la réponse spectrale pour cette longueur d'onde en calculant :

$$
R_{j}(\lambda)=\frac{\left\langle V_{\text {jecl. }}\right\rangle-\left\langle V_{\text {jobsc }}\right\rangle}{G \cdot \frac{\left\langle V_{\mathrm{ph}}\right\rangle}{\rho(\lambda)} T_{0}}
$$

où $G$ et $\rho(\lambda)$ sont respectivement le gain de la chaîne de mesure et le coefficient de calcul du flux réel reçu par le CCD au niveau du photoélément analysé.

\section{Résultats expérimentaux.}

On a porté sur la figure 7 quelques courbes de réponse spectrale relevées à des températures échelonnées entre $280 \mathrm{~K}$ et $80 \mathrm{~K}$. Les unités utilisées sont les $\mu \mathrm{m}$ pour l'axe horizontal et les (volts centimètre carré/ microjoules) pour l'axe vertical. On peut observer sur ces courbes des ondulations rapides; ces ondulations sont provoquées par les variations de transmission de la structure air-SiO ${ }_{2}$ située au-dessus de la surface supérieure du silicium actif (voir Fig. 2).

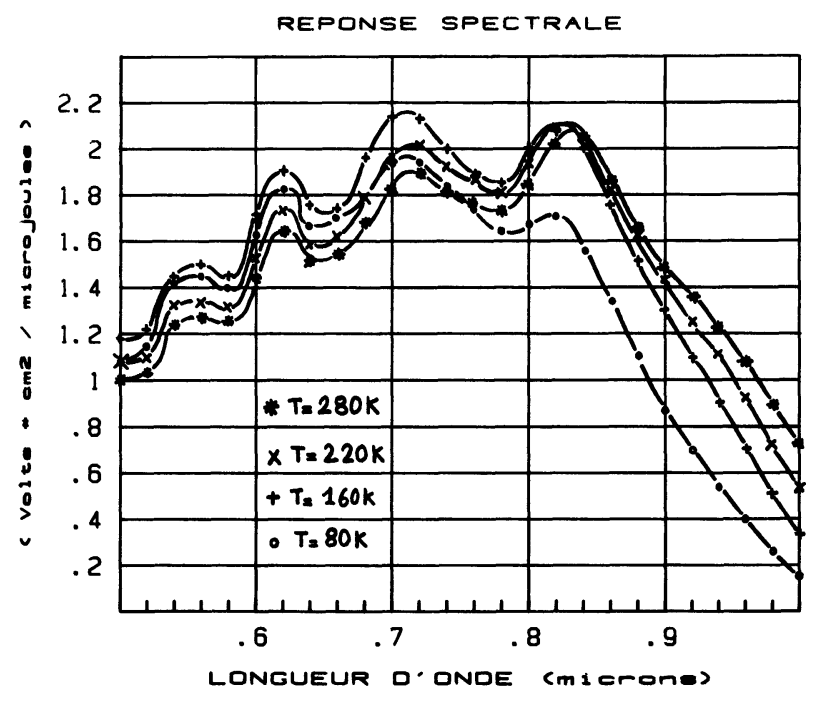

Fig. 7. - Courbes de réponse spectrale à différentes températures.

[Some spectral response curves.]

Ces variations de transmission sont liées à des interférences lumineuses se produisant dans les différentes couches traversées par le faisceau.

Van de Wiele (Réf. [7]) donne les évolutions de la transmission $t(\lambda)$ d'une telle structure. Le calcul est fait pour un faisceau de rayons parallèles et perpendiculaires aux plans séparant les différents milieux (air$\mathrm{SiO}_{2}-\mathrm{Si}$ ) : ce modèle, suffisant pour appréhender qualitativement les effets de $t(\lambda)$ sur la réponse spectrale, est cependant peu adapté à la description quantitative de l'amplitude des ondulations que nous observons. Ceci peut être lié, par exemple, à la présence d'un gradient d'indice dans la couche de $\mathrm{SiO}_{2}$, à la divergence du faisceau lumineux (demi-angle au sommet de l'ordre de $12^{\circ}$ ) et à la largeur de bande du 
faisceau en sortie du monochromateur. On notera cependant le bon accord entre les prévisions du modèle de la référence [7] et l'expérience pour ce qui est de la position des maxima et minima des ondulations (Fig. 8). On remarquera, de plus, que ces positions ne semblent pas dépendre de façon significative de la température.

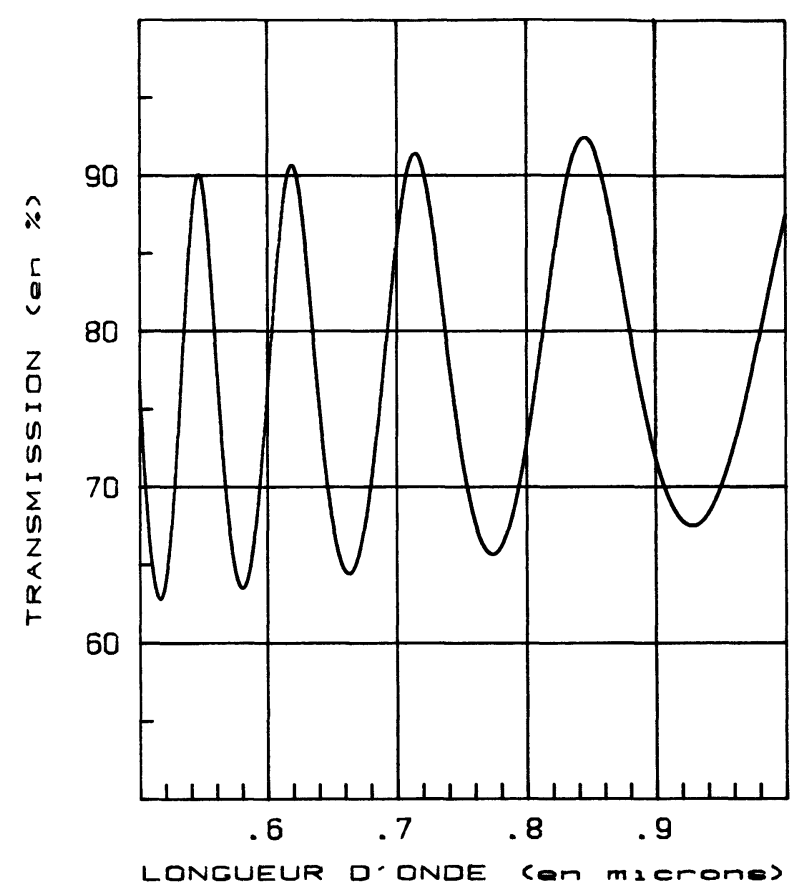

Fig. 8. - Courbe de transmission $t(\lambda)$ de la structure (Air, $\mathrm{SiO}_{2}, \mathrm{Si}$ ) calculée pour une épaisseur de $\mathrm{SiO}_{2}$ de $1,59 \mu \mathrm{m}$ à partir du modèle théorique proposé dans la référence [7].

[Theoretical transmission of the $\left(\mathrm{Air}-\mathrm{SiO}_{2}-\mathrm{Si}\right)$ system computed with the reference [7] model. This curve is computed for $\mathrm{a} \mathrm{SiO}_{2}$ layer thickness of $1.59 \mu \mathrm{m}$.]

\section{Méthode d'ajustement.}

Nous allons extraire des courbes de réponse spectrale expérimentales aux différentes températures les courbes de rendement interne pour les comparer aux prévisions du modèle théorique unidimensionnel.

Le but poursuivi est de déterminer les variations en fonction de la température des différents paramètres entrant dans le calcul du rendement interne théorique tel qu'il est défini par (6).

Dans la relation (3) reliant la réponse spectrale au rendement interne, deux quantités sont inconnues : il s'agit du coefficient $K$ et de la valeur de $t(\lambda)$. Pour éviter de faire intervenir ces deux quantités, nous définissons à partir des courbes $R_{j}(\lambda)$ aux différentes températures, des courbes $S_{j}(\lambda)$ passant par la valeur moyenne des ondulations de $R_{j}(\lambda)$ (voir Fig. 9). A partir des valeurs de $S_{j}(\lambda)$, on définit une fonction de $\lambda$ et de $T ; \sigma_{j}(\lambda, T)$ telle que :

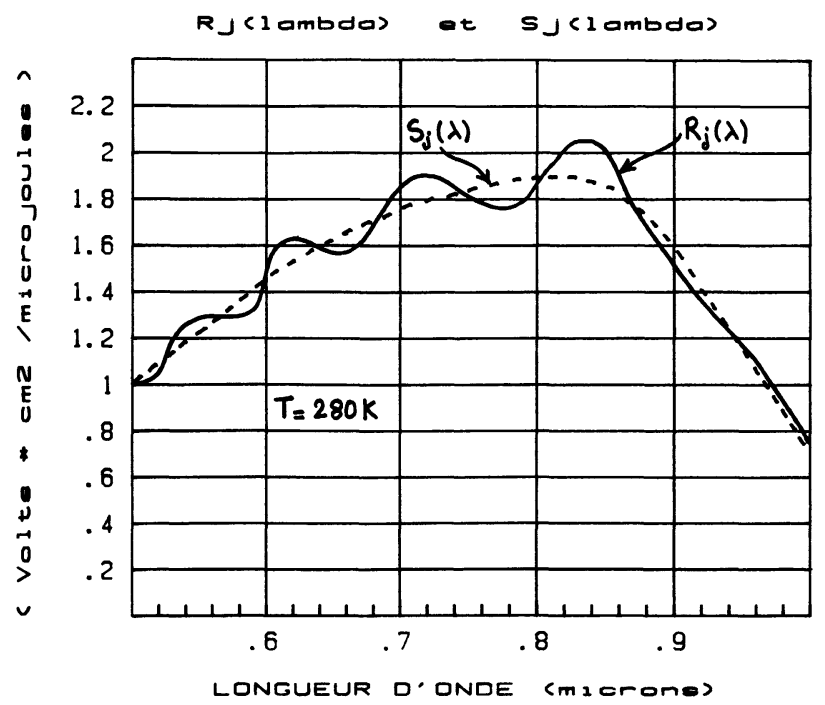

Fig. 9. - Exemple de courbes $R(\lambda)$ et $S(\lambda)$ à $280 \mathrm{~K}$.

$[R(\lambda)$ and $S(\lambda)$ curves at $280 \mathrm{~K}$.

$$
\sigma_{j}(\lambda, T)=\frac{\left(\frac{S_{j}(\lambda, T)}{\lambda}\right)}{\left(\frac{S_{j}(\lambda, T)}{\lambda}\right)_{\max }}
$$

$\sigma_{j}(\lambda, T)$ correspond au rendement quantique interne normalisé expérimental relatif au photoélément $\mathrm{n}^{\circ} j$. De même, à partir de la relation (6), on définit le rendement quantique interne normalisé théorique par la quantité :

$$
\Gamma(\lambda, T)=\frac{\eta_{\mathrm{i}}(\lambda, T)}{\left[\eta_{\mathrm{i}}(\lambda, T)\right]_{\max }}
$$

L'étape suivante consiste, à l'aide d'un logiciel approprié à chercher le jeu de $\left(L_{\mathrm{n}} ; L_{\mathrm{p}} ; L ; L_{\mathrm{s}} ; x_{j}\right)$ réalisant le meilleur ajustement des courbes $\sigma_{j}(\lambda, T)$ et $\Gamma(\lambda, T)$ à chaque température. Il est ensuite possible de tracer pour chaque température la courbe de rendement quantique interne $\eta_{i}(\lambda, T)$ du modèle unidimensionnel le mieux ajusté aux mesures (voir Fig. 10).

\section{Résultat des ajustements et interprétations.}

L'épaisseur $x_{j}$ de la zone avant a été estimée à $0,8 \mu \mathrm{m}$. Ceci rend les courbes de rendement interne peu dépendantes de la valeur du paramètre $L_{\mathrm{s}}$.

Les valeurs trouvées pour la longueur de diffusion des trous dans la zone $\mathrm{n}^{+}$aux températures de 280 et $78 \mathrm{~K}$ sont respectivement de $0,4 \mu \mathrm{m}$ et $0,1 \mu \mathrm{m}$. Ces valeurs sont en accord avec celles citées par Chamberlain (Réf. [9]). De plus, en formulant l'hypothèse que le modèle établi en 2.4 décrit correctement les variations du coefficient d'absorption aux tempéra- 


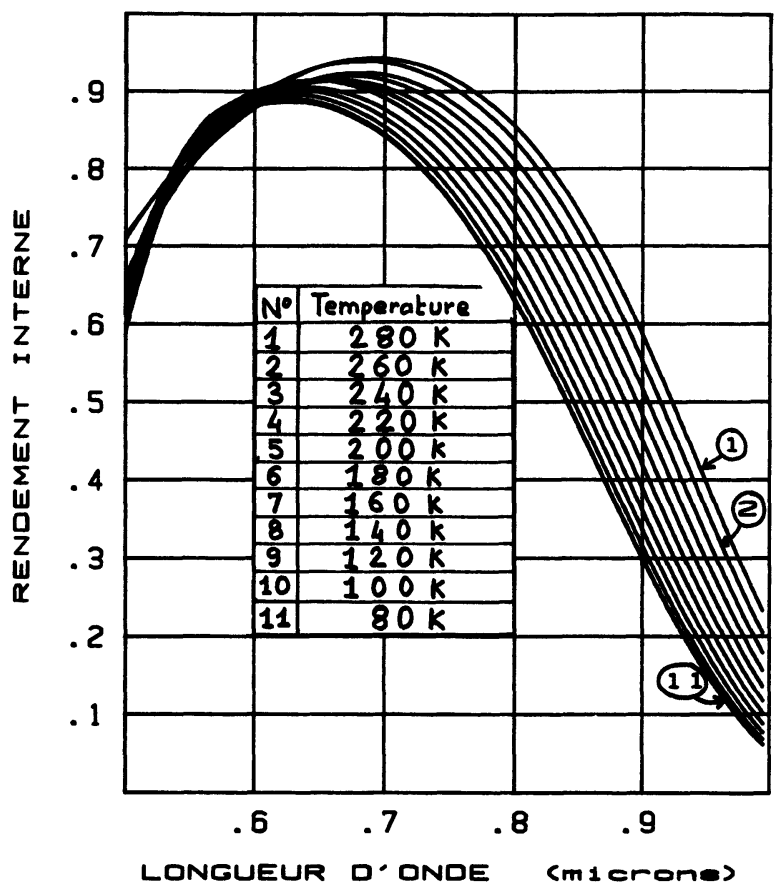

Fig. 10. - Rendement quantique interne théorique à différentes températures le mieux ajusté aux valeurs expérimentales.

[Internal quantum efficiency of the theoretical model which is in good fitting agreement for each temperature.]

tures intermédiaires, on est conduit à admettre que cette longueur de diffusion des trous $L_{\mathrm{p}}$ dans la zone $\mathrm{n}^{+}$ évolue suivant la courbe de la figure 11 .

A partir de l'ordre de grandeur des valeurs communément admises de longueurs de diffusion dans du silicium non dégénéré et au voisinage de la température ambiante (Réf. [4] page 167), on détermine au moment de l'ajustement de $\sigma(\lambda, 280)$ à $\Gamma(\lambda, 280)$ la valeur de $L$ (épaisseur totale de la zone active). On trouve $L \simeq 50 \mu \mathrm{m}$. Avec cette valeur de $L$, l'ajustement de $\sigma(\lambda, 78)$ à $\Gamma(\lambda, 78)$ montre que la longueur de diffusion des électrons de la partie $\mathbf{P}$ est supérieure à $\left(L-x_{\mathrm{p}}\right)$ même à la température de $78 \mathrm{~K}$.

Si on fait à nouveau l'hypothèse de la validité du modèle de $\alpha$ aux températures intermédiaires, les résultats des ajustements nous montrent que $L_{n}$ est toujours supérieur à $\left(L-x_{\mathrm{p}}\right)$ pour tout le domaine de température exploré et que les variations de $\alpha$ suffisent pour expliquer la déformation des courbes de rendement interne dans le rouge et l'infrarouge proche.

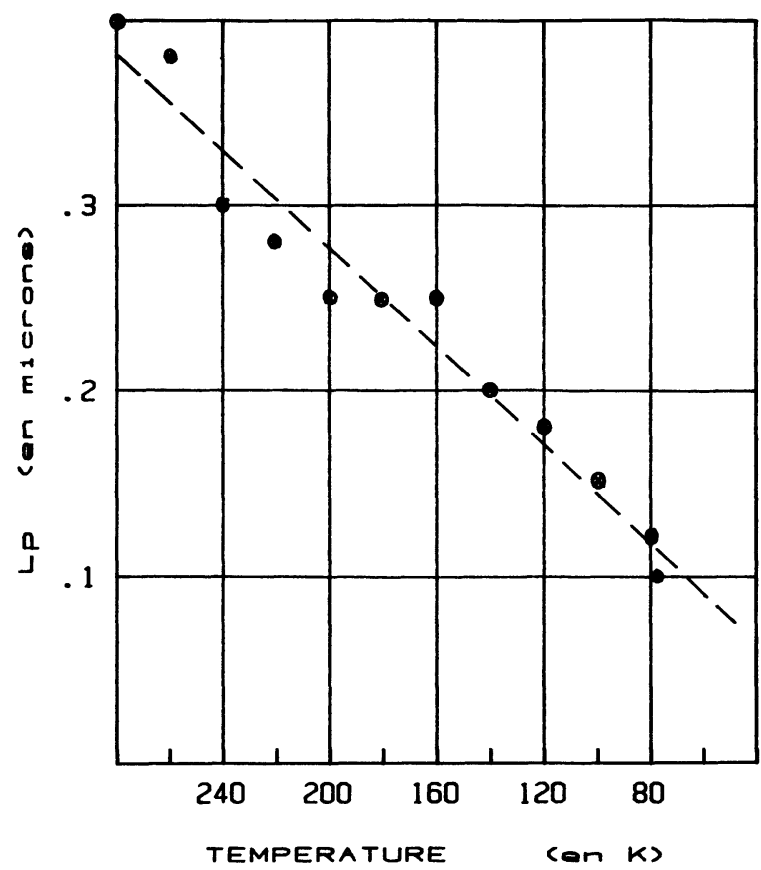

Fig. 11. - Evolution de la longueur de diffusion dans la zone avant avec la température.

[Diffusion length in the front layer versus temperature.]

\section{Conclusion.}

Nous avons effectué sur un détecteur CCD le relevé des courbes de réponse spectrale à des températures s'échelonnant entre $280 \mathrm{~K}$ et $78 \mathrm{~K}$. Les conditions expérimentales ont été choisies de façon telle que le modèle théorique du rendement interne d'un photoélément donné coïncide avec le modèle unidimensionnel d'une jonction plane supposée abrupte. L'utilisation des données expérimentales de Dash et Newman (Réf. [11]) nous a permis de dégager et d'évaluer les paramètres principaux qui régissent le comportement de la zone active aux températures voisines de $\mathbf{3 0 0}$ et $77 \mathrm{~K}$.

Dans l'hypothèse où le domaine d'adéquation de notre formule semi-empirique, permettant de recalculer les valeurs expérimentales de Dash et Newman (cf. $\S 2.4$ ), peut être étendu aux températures intermédiaires, on est conduit à admettre que les variations du rendement quantique interne du photoélément analysé sont dominées par les lois de dépendance du coefficient d'absorption et de la longueur de diffusion des trous dans le $\mathrm{n}^{+}$en fonction de la température (voir Fig. 4 et Fig. 11). 


\section{Bibliographie}

[1] Les dispositifs à transfert de charges, BERGER, BLAMOUTIER, COUtures, Descure, Thenoz, Rev. Tech. Thomson-Csf $12 \mathrm{n}^{\mathrm{o}} 1$ (1980).

[2] Charge Coupled Devices and Systems, Kosonocky, Zaininger, ChONG KI KIM, BURT, CaUchaN, HOZEMAN.

[3] Physics of Semiconductor devices, SM Sze Willey (2e Edition).

[4] Semiconductors and Semitals, Hovel, Solar Cells 11.

[5] Physique des dispositifs à semiconducteurs, VAPAILlE (Masson).

[6] Transport equations for the analysis of heavily doped Semiconductor Devices, LUNDSTROM, SchWARTZ, GrAY, Solid State Electron. 24 195-202.
[7] Proceedings of NATO Advanced Study Institute on Solid State Imaging, September 3-12, 1975, JeSPER, VAN DE WIELE, WHITE.

[8] A study of noise in charge coupled devices, Texas Instruments inc Dallas central Research Labs, May 75.

[9] New profiled silicon photodetector for improved short Wavelength efficiency, Chamberlain, J. Appl. Phys. 50 (1979) 7228-7231.

[10] Physique des semiconducteurs, KIREEV (Editions MIR).

[11] Intrinsic Optical Absorption in single-Crystal Germanium and Silicon at $77 \mathrm{~K}$ and $300 \mathrm{~K}$, DASH, W. C. and Newman, R., Phys. Rev. 99 № 4 (1955) 11511155. 